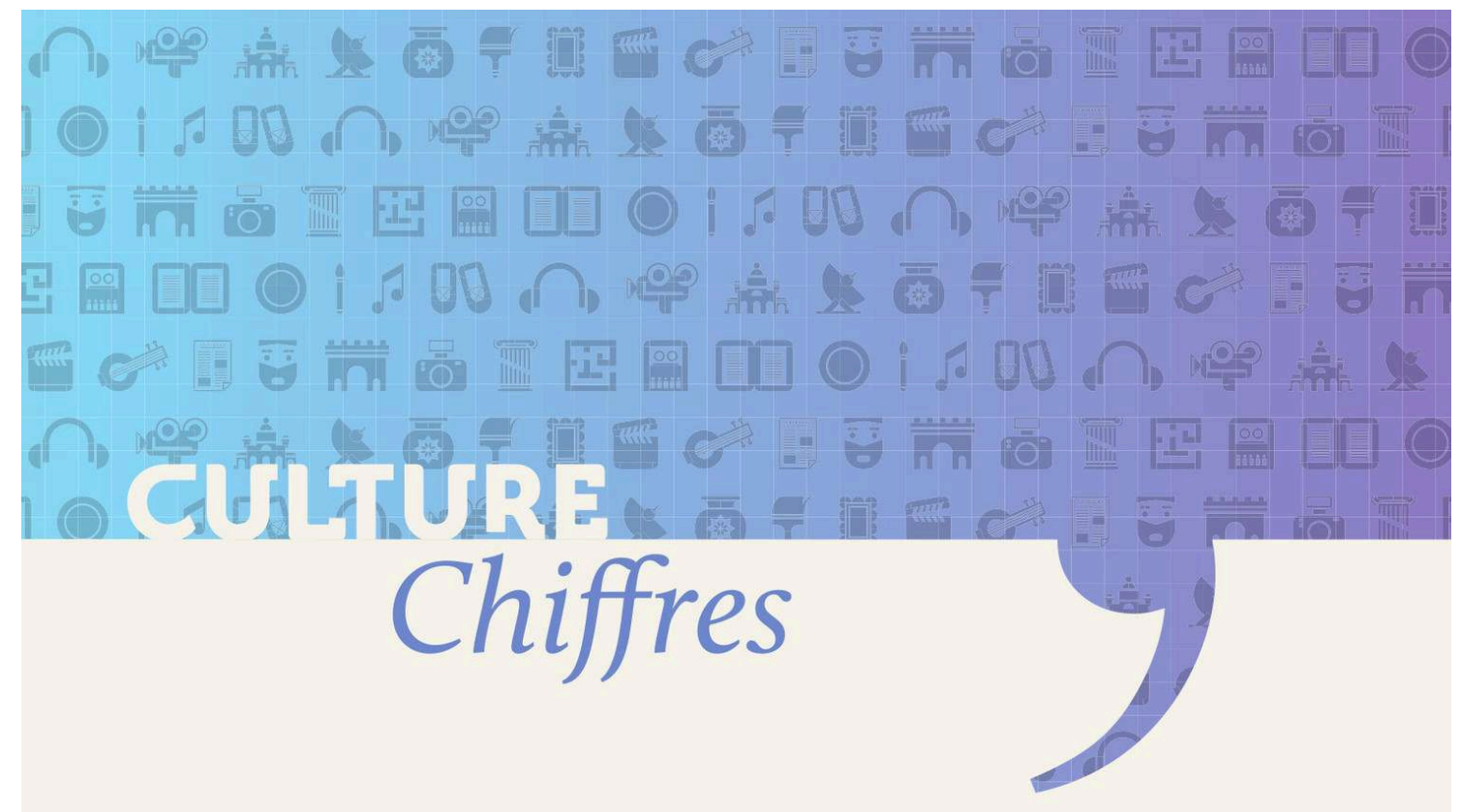

Auteurs du livre affiliés

à l'Agessa :

dégradation des perspectives

de revenus au fil des générations

Gwendoline Volat
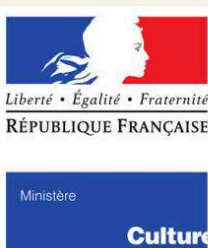

2016-2

Ministère de la Culture et de la Communication

Département des études,

de la prospective

et des statistiques 


\section{Auteurs du livre affiliés à l'Agessa : dégradation des perspectives de revenus au fil des générations}

AGESSA Writer Members: Historic Records Show Falling Income Prospects

\section{Gwendoline Volat}

Éditeur : Département des études, de la prospective et des statistiques

Lieu d'édition : Paris

Année d'édition : 2016

Date de mise en ligne : 23 février 2018

Collection : Culture chiffres

ISBN électronique : 9782111399419

\section{Sobooks}

http://books.openedition.org

Édition imprimée

Nombre de pages : 14

Référence électronique

VOLAT, Gwendoline. Auteurs du livre affiliés à l'Agessa : dégradation des perspectives de revenus au fil des générations. Nouvelle édition [en ligne]. Paris : Département des études, de la prospective et des statistiques, 2016 (généré le 25 avril 2021). Disponible sur Internet : <http://books.openedition.org/ deps/1194>. ISBN : 9782111399419 


\title{
Auteurs du livre affiliés à l'Agessa : \\ dégradation des perspectives de revenus au fil des générations
}

\author{
Gwendoline Volat
}

Depuis la fin des années 1970 et la création du régime social spécifique pour les auteurs de l'écrit, 11800 auteurs ont été affiliés au moins une fois à l'Agessa, l'organisme de gestion qui leur est dédié. En 2013, ils sont 5300 à être affiliés, soit 3,4 fois plus que trente ans plus tôt, un accroissement qui s'inscrit dans le mouvement général d'expansion des professions culturelles. Au sein des auteurs de l'écrit, on compte $47 \%$ d'écrivains, $31 \%$ d'illustrateurs et $22 \%$ de traducteurs en 2013. Ce sont ces deux dernières catégories d'auteurs qui ont particulièrement augmenté depuis la fin des années 1990. Les femmes sont de plus en plus présentes et représentent désormais la moitié des auteurs.

Depuis une quinzaine d'années, on entre dans le statut professionnel d'auteur de plus en plus tard et l'âge médian de première affiliation recule : il est de 39 ans en 2013 contre 34 ans en 1980. Pour autant, les durées d'affiliation s'allongent et passent de neuf ans pour la génération d'auteurs affiliés entre 1985 et 1989, à douze ans pour celle affiliée entre 1995 et 1999.

La population des auteurs de l'écrit tend à vieillir sur trente ans, un phénomène qui touche toutes les catégories d'auteurs : plus de la moitié des auteurs affiliés ont plus de 45 ans en 2013, alors que les 18-29 ans forment $4 \%$ seulement de l'ensemble de cette population. Si le revenu d'auteur progresse au fil des années d'affiliation, pour atteindre en médiane 25000 euros (avant impôts) après quinze ans d'affiliation, on observe que les perspectives de revenus se dégradent pour les auteurs affiliés depuis les années 2000. À durée d'affiliation égale, les générations plus jeunes d'auteurs déclarent des niveaux de revenus inférieurs à ceux de leurs aînés au même moment de leur carrière. En conséquence, les jeunes auteurs sont de plus en plus nombreux à ne pouvoir vivre que de la création et à cumuler différentes sources de revenus. Ces résultats pointent la nécessité d'une attention accrue pour la jeune création, afin de veiller au renouvellement de cette profession dans le contexte d'une dynamique économique défavorable. 


\section{Plus de 5200 auteurs affiliés en 2013 : des effectifs en hausse depuis 30 ans}

11800 auteurs du livre ${ }^{1}$ ont été affiliés au moins une fois à l'Agessa entre 1979 et 2013 (voir «Définition de source : la base de données individuelles de l'Agessa », p. 10).

En 1979, année de création de ce régime social spécifique, 1550 auteurs ont été affiliés. Puis, en moyenne, 300 nouveaux auteurs ont été affiliés chaque année tandis que 210 auteurs sortaient de l'affiliation, soit un flux net moyen de 90 auteurs par an. Le nombre d'affiliés a été multiplié par plus de trois en trente-cinq ans pour atteindre 2590 affiliés en 1993, puis 3310 en 2001 et enfin 5280 en $2013^{2}$ (graphique 1). Cet accroissement s'inscrit dans le mouvement général d'expansion des professions culturelles depuis vingt ans (+ $50 \%$ entre 1991 et $2011^{3}$ ). D'autres professions culturelles, cependant, liées aux arts graphiques, à la mode et à la décoration ou aux métiers du spectacle, ont connu une croissance plus importante.

Bien que leur part relative ait diminué au cours du temps, les écrivains (y compris auteurs d'œuvres dramatiques) composent, quelle que soit l'année, la part la plus importante des auteurs du livre affiliés (47\% de l'ensemble des affiliés du livre en 2013), suivis des illustrateurs (31\%) et des traducteurs (22\%) (graphique 1).

\section{Graphique 1 - Évolution de la population des auteurs selon leur catégorie, 1979-2013}

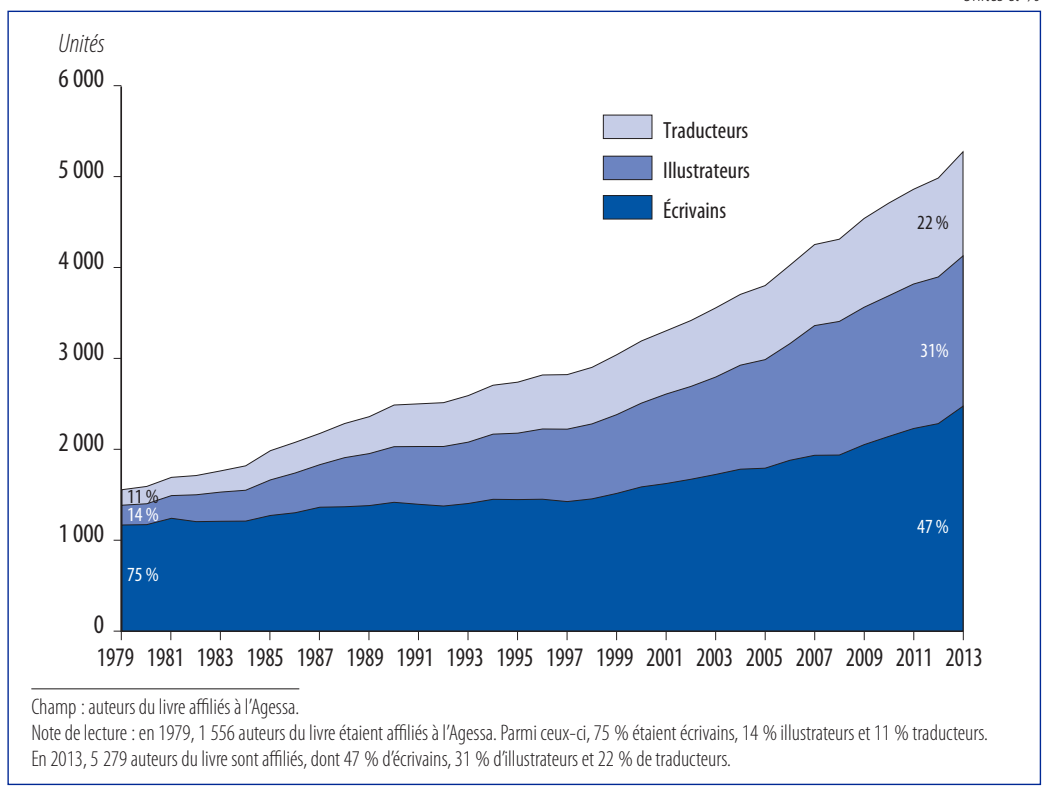

Source : Agessa/DEPS, Ministère de la Culture et de la Communication, 2016

1. Les auteurs du livre désignent ici les écrivains, les traducteurs, les illustrateurs (du livre, hors presse) ainsi que les dramaturges. On dénombre 324 dramaturges affliés au moins une fois entre 1979 et 2013, qui sont comptés avec les écrivains dans la suite de l'étude.

2. Le seuil d'affiliation était de 1200 fois la valeur horaire du Smic en 1993, il a été abaissé en 2001 à 900 fois la valeur horaire du Smic, sans que cela ait eu un impact mécanique sur l'évolution du nombre d'affiliés auteurs du livre.

3. Marie Gouron, Frédérique PATuREAU, Vingt ans d'évolution de l'emploi dans les professions culturelles, 1991-2011, Ministère de la Culture et de la Communication, DEPS, coll. « Culture chiffres», 2014-6, 2014. 


\section{Un début d'affiliation plus tardif depuis le milieu des années 2000}

Regroupés par année de première affiliation, les auteurs constituent plusieurs " générations » dont les caractéristiques peuvent varier. Pour l'ensemble des auteurs affiliés entre 1980 et 2004, l'âge médian en début d'affiliation a été constant : la moitié des auteurs de ces générations avaient 34 ans ou plus lorsqu'ils ont déclaré leurs premiers revenus d'auteur (graphique 2 ).

Les nouvelles générations d'auteurs, soit ceux affiliés après 2005, déclarent leurs premiers revenus d'auteur à un âge de plus en plus avancé : la moitié des auteurs des années 2010-2013 sont âgés de 39 ans ou plus au moment de leur première affiliation (graphique 2).

L'avancée de l'âge médian lors de la première affiliation concerne l'ensemble des catégories d'auteurs, bien qu'on ne s'affilie pas au même âge selon que l'on est écrivain, traducteur ou illustrateur. Les illustrateurs sont les plus jeunes à s'affilier : un illustrateur sur deux a 33 ans ou plus lorsqu'il déclare ses premiers revenus dans les années 2010. L'âge médian de première affiliation des traducteurs (37 ans) est proche de celui de l'ensemble. Enfin, les écrivains sont les plus âgés à s'affilier : I'âge médian de première affiliation est de 43 ans (graphique 2).

\section{Graphique 2 - Âge médian lors de la première affiliation selon la génération et la catégorie d'auteur, 1980-2013}

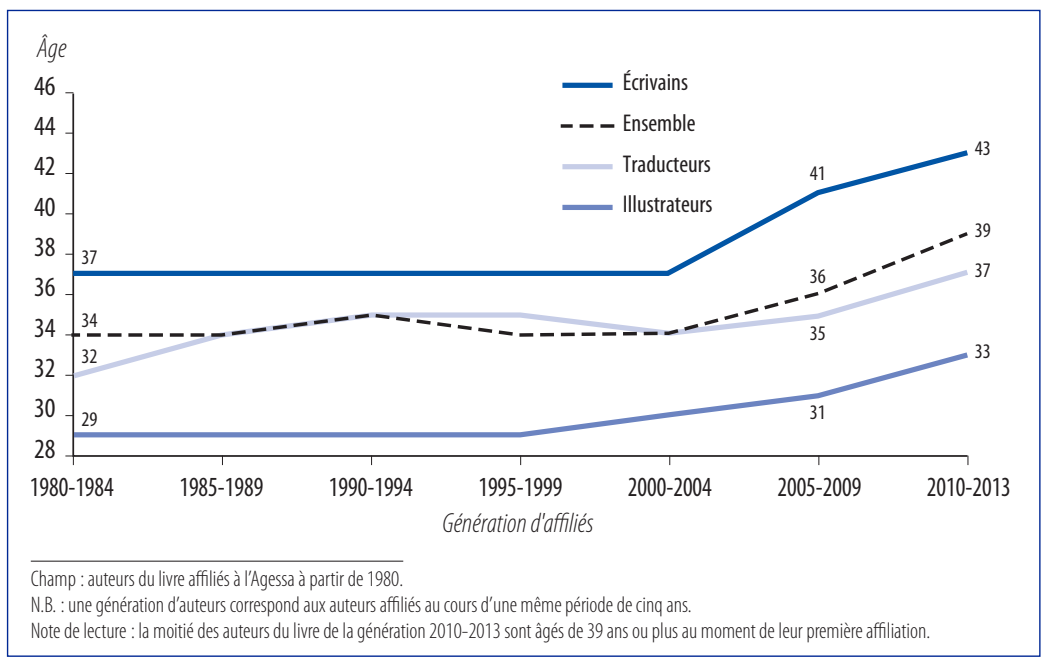

Source: Agessa/DEPS, Ministère de la Culture et de la Communication, 2016

\section{Des durées d'affiliation plus longues}

La durée d'affiliation d'un auteur correspond au nombre d'années comprises entre la première et la dernière année de déclaration de revenus, y compris les éventuelles années d'interruption d'activité. La durée d'affiliation médiane s'est légèrement allongée à partir du milieu des années 1980, passant de neuf ans pour la génération d'auteurs affiliés entre 1985 et 1989 à douze ans pour la génération affiliée entre 1995 et 1999 (graphique 3). 
Graphique 3 - Durée médiane d'affiliation selon la génération et la catégorie d'auteur, 1980-2004

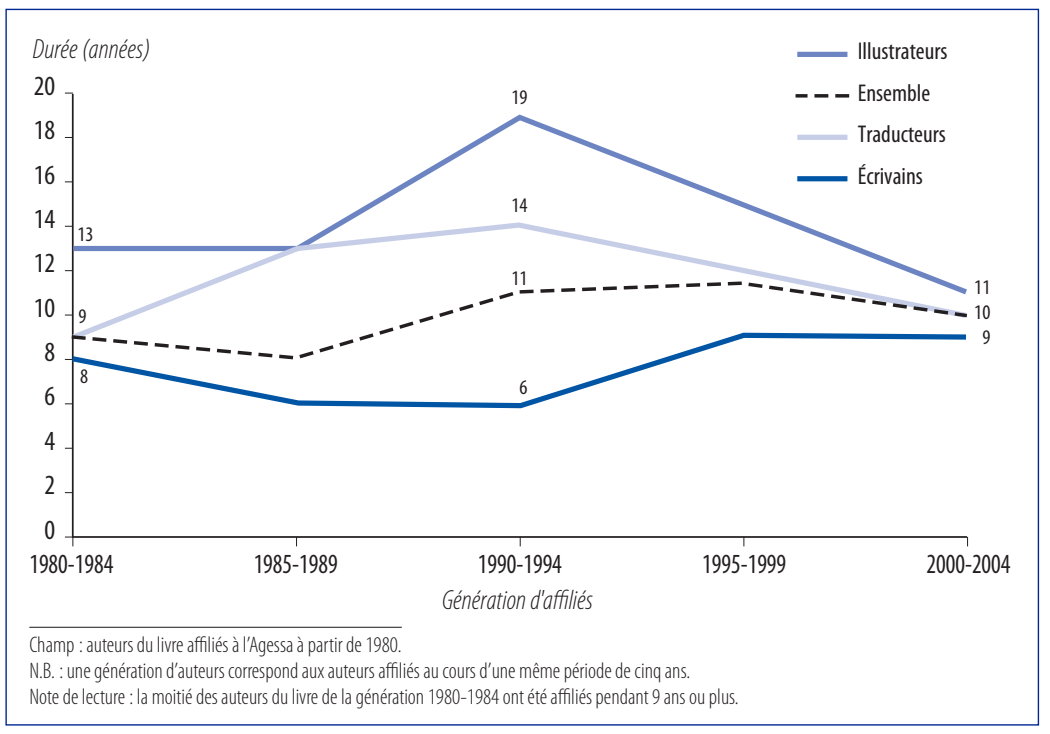

Source : Agessa/DEPS, Ministère de la Culture et de la Communication, 2016

\section{Graphique 4 - Part des auteurs ayant une durée d'affiliation inférieure ou égale à trois ans selon la génération et la catégorie, 1980-2004}

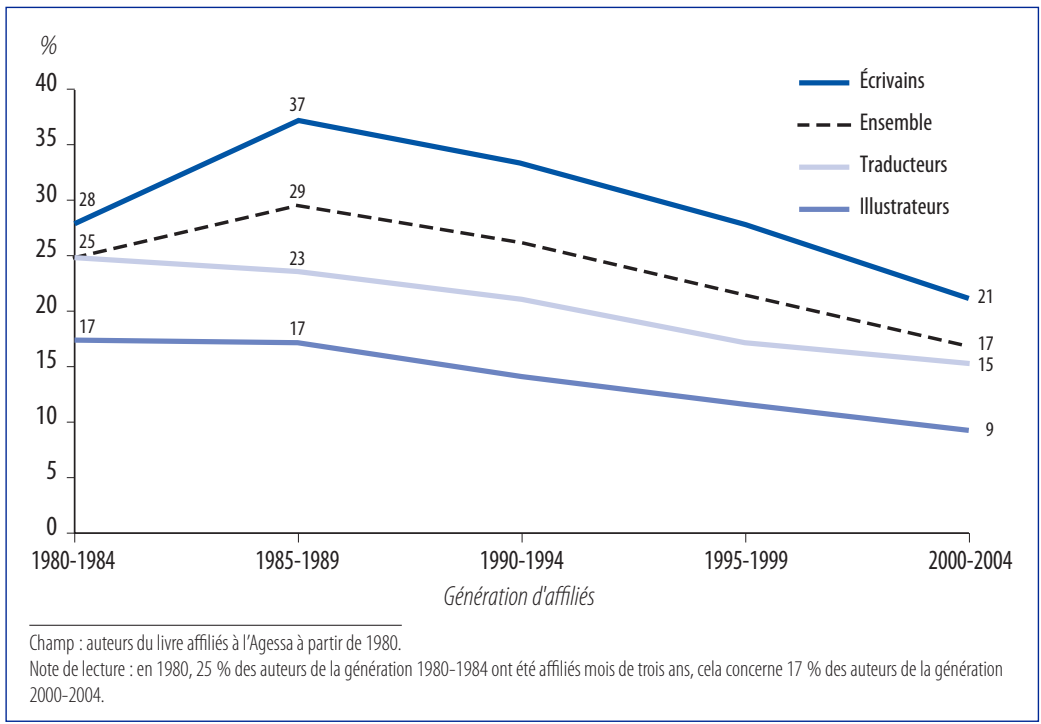

Source: Agessa/DEPS, Ministère de la Culture et de la Communication, 2016 
Cette évolution s'explique par un recul des courtes périodes d'affiliation (de une à trois années) (graphique 4). Ainsi, alors que $8 \%$ des auteurs de la génération 19801984 n'ont été affiliés qu'une seule année, cela concerne seulement $2 \%$ des auteurs de la génération 2000-2004.

Quelle que soit la génération d'auteurs, les illustrateurs et, dans une moindre mesure, les traducteurs ont une période d'affiliation plus longue que les écrivains.

Les auteurs du livre sont peu concernés par les interruptions d'affiliation: sur l'ensemble des affiliés après 1980, moins d'un sur dix a connu une interruption d'affiliation avec une reprise ultérieure et ce, quelle que soit la catégorie d'auteur. Parmi ceux-ci, le nombre d'années d'interruption médian est de trois années.

\section{Une population d'auteurs qui vieillit...}

En trente ans, la moyenne d'âge des auteurs est passée de 36 ans en 1980 à 47 ans en 2013, ce qui s'explique par un double mouvement ; la part des jeunes auteurs, soit ceux âgés de 18 à 29 ans, décroît tandis que celle des 45 ans et plus augmente fortement (respectivement -26 points et +32 points). En 2013, plus de la moitié des auteurs sont âgés de plus de 45 ans alors que les jeunes auteurs (18 à 29 ans) représentent seulement $4 \%$ de la population (graphique 5 ).

L'ensemble des professions connaît le même phénomène de vieillissement mais les écrivains sont toujours plus âgés que les traducteurs et les illustrateurs : en 2013, ils ont en moyenne 49 ans, contre 47 ans pour les traducteurs et 43 ans pour les illustrateurs.

Graphique 5 - Part de la population des auteurs selon la tranche d'âge, 1980-2013

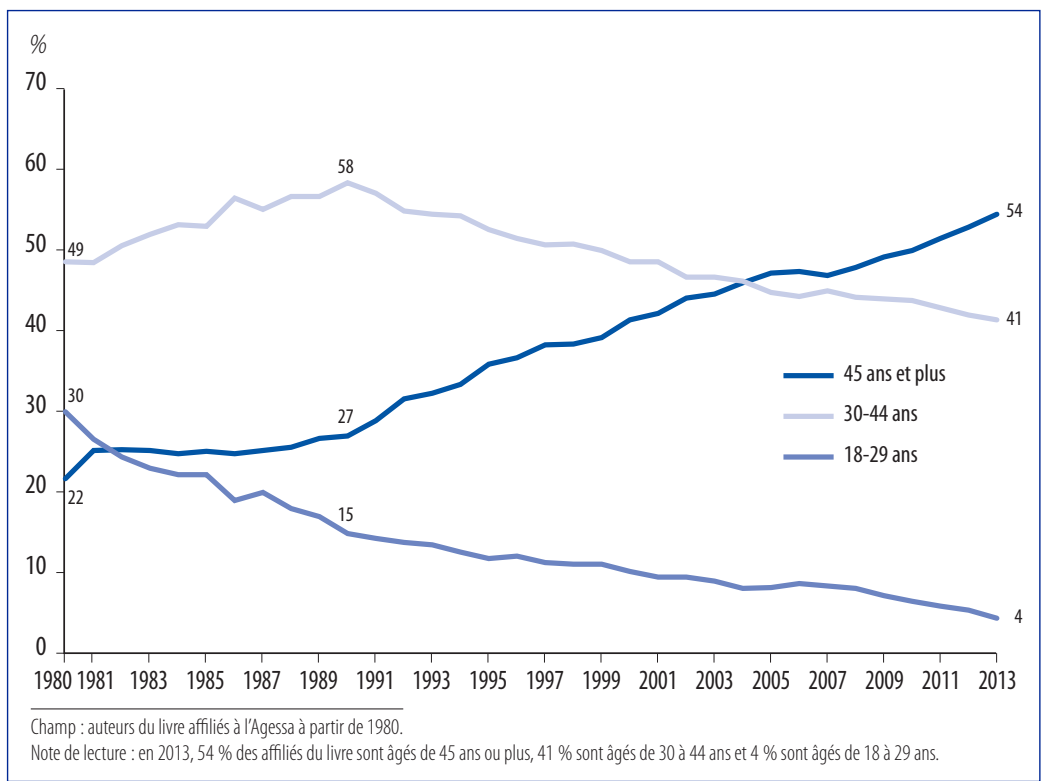




\section{...et se féminise}

La part de femmes au sein des auteurs du livre a progressé depuis trente ans: en 1980, 40 \% des auteurs affiliés étaient des femmes, elles représentent désormais la moitié des auteurs. Cette évolution s'explique plus particulièrement par l'augmentation de la part des femmes au sein des écrivains (de $30 \%$ à $50 \%$ ).

Au contraire de la tendance observée chez les écrivains, la part d'illustratrices est restée stable jusque dans les années 1990, puis elle a commencé à décliner (de $38 \%$ à $31 \%$ ) jusqu'au milieu des années 2000, pour s'établir à nouveau à $38 \%$, en raison des nouvelles générations, particulièrement féminisées (graphique 6).

Enfin, quelle que soit l'année, la population des traducteurs et traductrices est la plus féminisée : près de sept traducteurs sur dix sont des femmes.

\section{Graphique 6 - Part des femmes selon la catégorie d'auteur, 1980-2013}

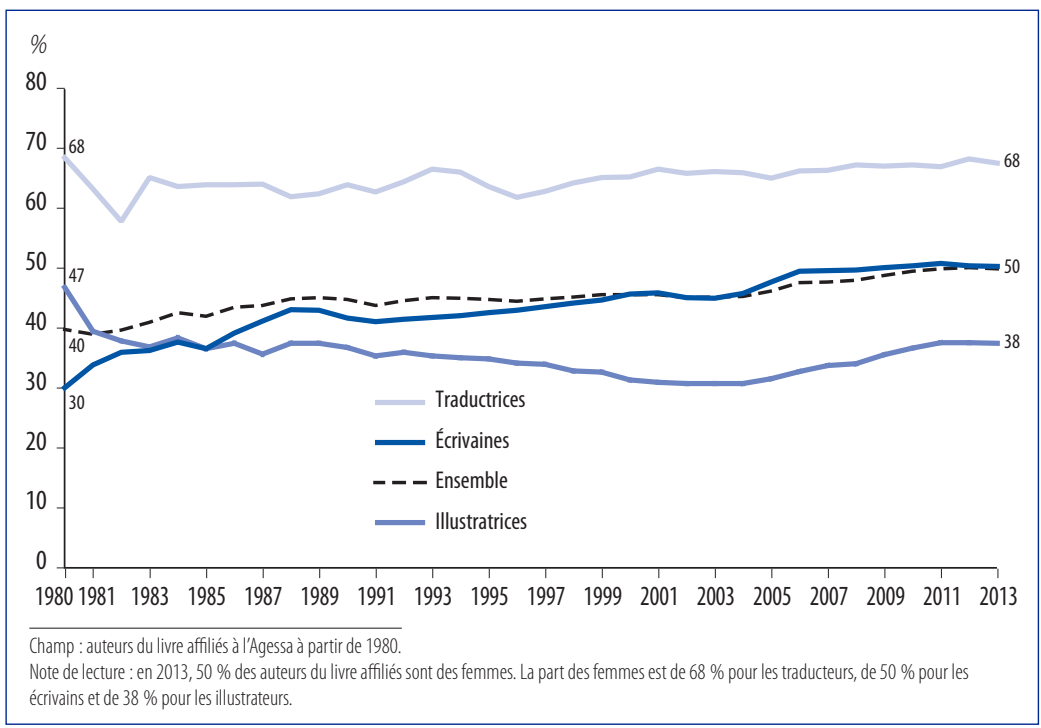

Source: Agessa/DEPS, Ministère de la Culture et de la Communication, 2016

\section{Le revenu d'auteur progresse au fil des années d'affiliation}

Les revenus augmentent avec la durée d'affiliation. Ainsi, la première année d'affiliation, un auteur sur deux déclare 9000 euros de droits d'auteur annuels. Le revenu médian augmente rapidement au cours des premières années puisqu'il double après quatre années d'affiliation. Ensuite, il continue de progresser à un rythme plus faible ( $+2 \%$ par an) pour atteindre un maximum de 27020 euros au bout de vingt ans de carrière (graphique 7).

Les premiers revenus médians sont différents selon les catégories : de 9730 euros pour les écrivains, 8560 euros pour les illustrateurs à 7900 euros pour les traducteurs. Au cours des dix premières années d'affiliation, le taux de croissance annuel du revenu médian des illustrateurs et des traducteurs est plus élevé (+10\% par an) que celui des écrivains (+9\%). Ainsi, les revenus d'auteur médians après quinze ans d'affiliation atteignent environ $25000 €$, quelle que soit la catégorie (graphique 7). 


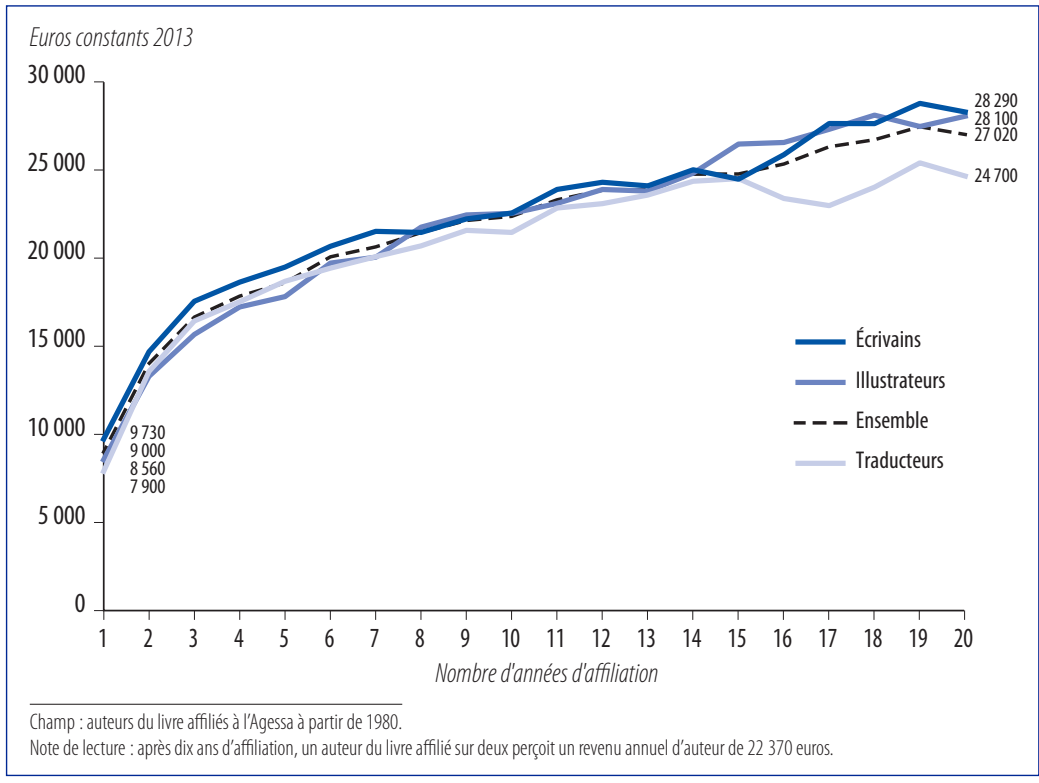

Source: Agessa/DEPS, Ministère de la Culture et de la Communication, 2016

Les femmes, quel que soit le type d'activité exercée en tant qu'auteure du livre, ont des revenus moins élevés que les hommes, et l'écart de revenu médian se creuse au fil des années d'affiliation. La première année, une auteure du livre sur deux déclare 8010 euros de revenus d'auteur, soit $21 \%$ de moins que son homologue masculin. Après vingt ans d'affiliation, cet écart est de 30 \% (21 960 euros pour les femmes contre 31420 euros pour les hommes). L'écart est particulièrement marqué parmi les illustrateurs et illustratrices : de $23 \%$ la première année d'affiliation, il atteint $41 \%$ après vingt ans de carrière.

\section{De moins en moins d'auteurs déclarent uniquement des revenus d'auteur}

Les revenus d'un auteur ne sont pas composés uniquement de droits d'auteur: celui-ci peut exercer une activité salariée ou au titre d'indépendant dans d'autres domaines, parallèlement à son activité de création. La part d'auteurs déclarant exclusivement des droits d'auteur progresse avec le nombre d'années d'affiliation: plus d'un auteur sur deux ne déclare pas de revenus complémentaires lors de la première année d'affiliation, ils sont sept sur dix à le faire après vingt ans de carrière.

Cependant, depuis le début des années 2000, la part d'auteurs du livre affiliés déclarant exclusivement des revenus d'auteur diminue progressivement et ne concerne plus que $54 \%$ des auteurs, contre $70 \%$ quinze ans plus tôt. Cette tendance concerne l'ensemble des générations, et plus particulièrement les auteurs affiliés après 2000. 
Les auteurs n'exerçant pas d'activité complémentaire déclarent un revenu d'auteur médian sensiblement plus élevé (20 860 euros par an) que ceux qui cumulent plusieurs activités (14 210 et 17140 euros selon le type de revenu complémentaire) (tableau 1).

En 2013, en revanche, le fait de cumuler plusieurs activités assure un revenu total supérieur aux seuls revenus artistiques. Un auteur qui ne tire ses ressources que de la création a des revenus totaux inférieurs à ceux qui cumulent création et activité indépendante ou salariée (respectivement - $19 \%$ et - $26 \%$ ). Un peu moins d'un auteur affilié sur dix ( $8 \%$ ) cumule toutes les activités. Ce sont ces auteurs qui perçoivent le revenu total annuel médian le plus élevé (33 150 euros par an).

Tableau 1 - Revenus médians selon la composition des revenus en 2013

\begin{tabular}{|c|c|c|c|c|c|}
\hline Situation & $\begin{array}{l}\text { Part } \\
\text { (\%) }\end{array}$ & $\begin{array}{l}\text { Revenu } \\
\text { d'auteur }\end{array}$ & $\begin{array}{c}\text { Revenu } \\
\text { d'indépendant }\end{array}$ & $\begin{array}{l}\text { Salaire non } \\
\text { artistique }\end{array}$ & Total \\
\hline $\begin{array}{l}\text { Auteur ne déclarant } \\
\text { que des revenus d'auteur }\end{array}$ & 54 & 20860 & - & - & 21080 \\
\hline $\begin{array}{l}\text { Auteur déclarant } \\
\text { des revenus salariés }\end{array}$ & 28 & 15460 & - & 4640 & 26050 \\
\hline $\begin{array}{l}\text { Auteur déclarant } \\
\text { des revenus d'indépendant }\end{array}$ & 10 & 17140 & 5220 & - & 28714 \\
\hline $\begin{array}{l}\text { Auteur déclarant des revenus } \\
\text { salariés et d'indépendant }\end{array}$ & 8 & 14210 & 4910 & 3250 & 33150 \\
\hline
\end{tabular}

Source: Agessa/DEPS, Ministère de la Culture et de la Communication, 2016

\section{Une réduction sensible de la concentration des revenus d'auteur jusqu'en 2000}

La concentration des revenus peut être approchée par l'indice de Gini : plus celuici est proche de 1, plus la répartition des revenus d'auteur est inégalitaire. De 1979 à 2000 , la répartition des revenus d'auteur a été de plus en plus égalitaire entre tous les affiliés, l'indice passant de 0,65 à 0,57 en 2000. Ainsi, en 1979, les $30 \%$ d'auteurs les mieux rémunérés concentraient $78 \%$ des revenus d'auteur, tandis qu'en 2000 , ils en concentraient $71 \%$. Depuis 2000, l'indice de Gini oscille entre 0,56 et 0,60 et s'établit à 0,56 en 2013.

En 2013, $1 \%$ des auteurs les mieux rémunérés (revenus d'auteurs supérieurs à 306870 euros par an) se partagent $20 \%$ des revenus d'auteur. Ils sont en moyenne âgés de 52 ans et ont déclaré en moyenne 16 années de revenus. Il s'agit essentiellement d'écrivains (71\%) résidant à Paris (57\%). Peu de femmes sont présentes (28\%) parmi les 46 auteurs les mieux rémunérés en 2013. 


\section{Des perspectives de revenus moindres pour les jeunes générations d'auteurs}

Selon leur année d'affiliation, les auteurs ont des perspectives d'évolution de revenus différentes. Bien que les revenus d'auteur médians des premières années soient relativement similaires d'une génération d'auteurs à l'autre, les nouvelles générations (auteurs affiliés après 2000) ne connaissent pas la même croissance de revenus que les générations précédentes. Alors qu'un auteur sur deux de la génération 19801984 déclare 23970 euros de revenus d'auteur après dix ans d'affiliation, un auteur sur deux de la génération 2000-2004 déclare 19870 euros, soit $17 \%$ de moins (graphique 8).

Ces effets de générations sont propres aux écrivains et aux illustrateurs, et les écarts sont particulièrement marqués pour les illustrateurs: les revenus d'auteurs médians de la génération 1980-1984 ont progressé en moyenne de $14 \%$ par an entre la première et la dixième année de revenu, alors que la progression n'est que de $10 \%$ par an pour les illustrateurs affiliés entre 2000 et 2004. Ces effets générationnels ne s'observent pas, en revanche, pour la population des traducteurs.

\section{Graphique 8 - Évolution du revenu d'auteur médian selon le nombre d'années d'affiliation et la génération d'auteurs}

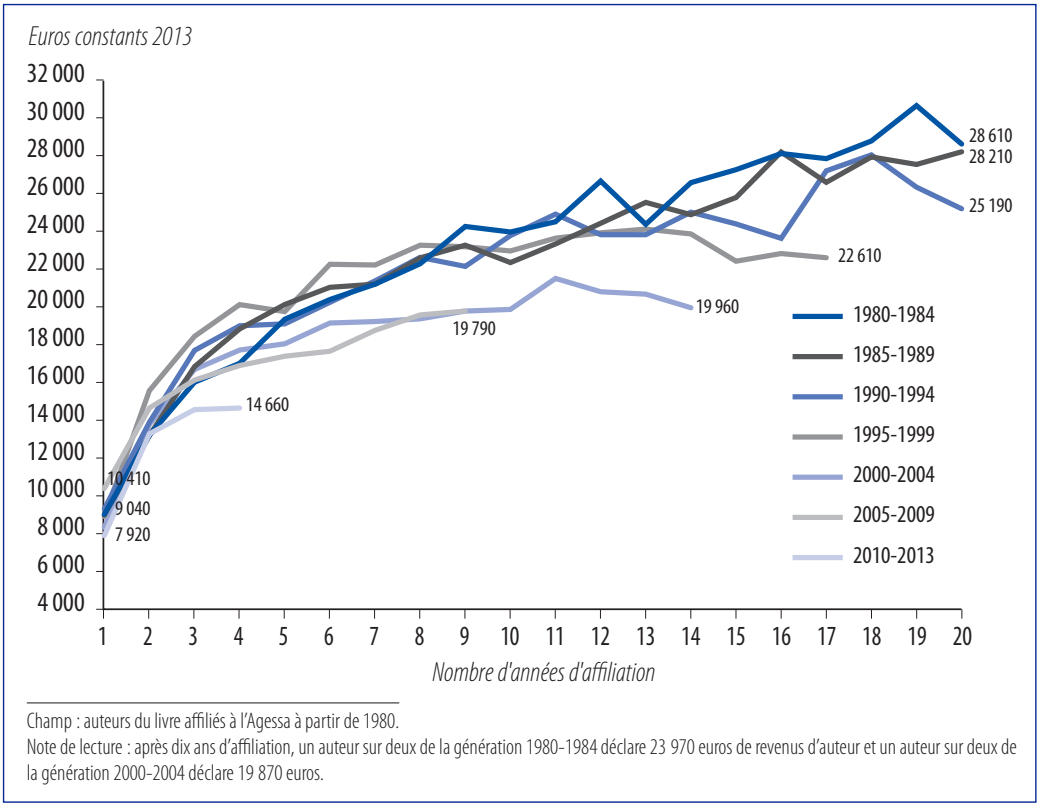

Source: Agessa/DEPS, Ministère de la Culture et de la Communication, 2016 


\section{Définition et méthodes}

Revenus d'auteur : rémunérations annuelles issues de la création déclarées en traitements et salaires et/ou en chiffres d'affaires, bruts (avant déduction des charges sociales et impôts), en euros constants 2013. Les revenus d'auteur ont été déflatés à l'aide de l'indice des prix à la consommation.

En raison de données manquantes, les chiffres d'affaires ne sont pas renseignés pour la période 1994 à 2000, ils ont été estimés sur la base des chiffres d'affaires déclarés à partir de 2001. Cette correction concerne essentiellement les illustrateurs qui privilégient davantage les modes « déclaration contrôlée » ou «micro-BNC » pour leur déclaration de revenus (70\% des illustrateurs affiliés en 2013).

Médiane : si on ordonne la distribution d'une variable (revenus d'auteur, âge à l'affiliation, durée d'affiliation), la médiane est la valeur qui partage cette distribution en deux parties égales. Ainsi, pour les revenus d'auteur, la médiane est le revenu d'auteur au-dessous duquel se situent $50 \%$ des auteurs. C'est de manière équivalente le revenu d'auteur audessus duquel se situent $50 \%$ des auteurs.

La médiane est préférée à la moyenne qui est tirée vers le haut par les revenus d'auteurs très élevés, même s'ils sont peu nombreux. C'est pour cette raison qu'elle est généralement supérieure à la médiane. Par ailleurs, l'incertitude qui affecte les valeurs extrêmes, en particulier les valeurs élevées, se reporte sur la moyenne mais n'affecte pas la médiane. Celle-ci est de ce point de vue un indicateur plus fiable.

\section{Encadré 1}

\section{Définition de source : la base des données individuelles de l'Agessa}

Toute diffusion ou exploitation commerciale par un diffuseur d'une œuvre originale d'un artiste auteur donne lieu au versement d'une rémunération (les droits d'auteur). Lorsque l'artiste auteur exerce son activité de façon indépendante (non salariée) et qu'il réside fiscalement en France, le versement des droits d'auteur par le diffuseur ou la vente d'œuvres s'accompagnent du prélèvement de cotisations et contributions obligatoires, opération appelée précompte. Ces cotisations et contributions sont collectées par l'Association pour la gestion de la sécurité sociale des auteurs (Agessa) pour les activités de création littéraire.

Les cotisations aux assurances sociales (vieillesse, maladie, maternité, invalidité, décès), la CSG et la CRDS sont dues par l'artiste auteur au titre du régime de protection sociale des artistes auteurs (articles L.382-1 à L.382-14 et R.382-1 à R.382-36 du Code de la sécurité sociale); ce régime spécifique est rattaché au régime général des salariés. Sous conditions de ressources et s'il en fait la demande, l'artiste auteur est affilié à l'Agessa; à ce titre, il bénéficie des prestations équivalentes à celles du régime général (indemnités journalières, congés maternité, etc.). Pour cela, il doit déclarer une assiette sociale (traitements et salaires et/ou bénéfices non commerciaux majorés de 15 \%) supérieure au seuil d'affiliation. Lorsque le seuil d'affiliation n'est pas atteint, l'affiliation ou son maintien peut être prononcé, à titre dérogatoire, par la commission professionnelle de l'Agessa, qui juge de l'engagement professionnel de l'artiste auteur. Le seuil d'affiliation a été modifié au cours du temps: de 1200 fois la valeur du Smic horaire avant 2001, il est passé à 900 fois la valeur du Smic horaire (8 $577 €$ en 2014).

La base de l'Agessa contient les données individuelles des auteurs ayant été affiliés au moins une année à ce régime de sécurité sociale. La table relative aux auteurs du livre (écrivains, dramaturges, illustrateurs et traducteurs) contient 115548 lignes. Une observation correspond aux revenus déclarés par un auteur pour une année donnée. Les situa- 
tions de non-affiliation, notamment les refus d'affiliation après examen de la commission professionnelle, sont exclus de l'analyse. Les revenus d'auteur nuls ont également été exclus de l'analyse.

Ces deux traitements suppriment $9 \%$ de la table, passant de 12086 à 11787 identifiants (2,5\%). Plus d'un auteur supprimé sur deux a été affilié avant 1980, II s'agit d'anciens enregistrements dont la suppression n'impacte pas les analyses.

\section{Effet de troncature à gauche}

L'Agessa ayant été créée en 1979, la table enregistre les années de «fin de période d'affiliation » pour certains auteurs. Ainsi, pour étudier les parcours d'affiliation des auteurs, on restreint l'analyse aux auteurs affiliés après 1980 (inclus). Si l'on restreint à ce champ spécifique, 10114 auteurs sont conservés.

\section{Encadré 2}

\section{La situation économique du secteur du livre}

Contrairement au secteur de la presse dont l'activité économique est en recul depuis les années 2000, le secteur du livre n’a pas été aussi fortement affecté par la dématérialisation des contenus. Ainsi, d'après les comptes nationaux de l'Insee, la valeur ajoutée du secteur du livre (codes NAF librairies [47.61Z], édition [58.11Z] et activités de traduction et d'interprétariat [74.30Z]) a progressé de $30 \%$ entre 1995 et 2004 pour s'établir à 2,7 milliards d'euros courants. Elle est restée à ce niveau jusqu'en 2012 et décline légèrement depuis, pour s'établir à 2,6 milliards en 2014.

D'après les comptes nationaux, la consommation de livres, quels que soient le support et le format, en volume (corrigé de l'inflation) est en baisse depuis 2010 (- 9,3\%) et s'établit à 3,6 milliards d'euros.

Les écrivains, traducteurs et illustrateurs, qu'ils soient affiliés ou non à l'Agessa, sont approchés par les effectifs de l'enquête emploi. Entre 2004 et 2013, le nombre d'auteurs littéraires est relativement constant sur la période (11 300 individus). Cependant, cette catégorie englobe également les dialoguistes et les paroliers. Le nombre de traducteurs et interprètes a augmenté de 60 \% entre 2004 et 2013, la plus forte hausse ayant eu lieu en 2005. 


\section{Abstract}

\section{AGESSA Writer Members: Historic Records Show Falling Income Prospects}

Since the late 1970s when the French-government-run authors' social security agency AGESSA was set up, 11,800 writers have been affiliated with it at least once. In 2013, it had 5,300 affiliates, 3.4 times more than the figure 30 years earlier, a rise which is indicative of the overall growth of the cultural professions. In 2013, the authors category included 47\% writers, 31\% illustrators and 22\% translators. The latter two groups have grown particularly since the late 1990s. Women form a growing proportion of its affiliates and now represent half of all authors.

Over the last fifteen years, people have been joining the ranks of authors increasingly later in life, with the median age of first affiliation rising: age 39 in 2013 as compared with 34 in 1980. Nevertheless, people now are being affiliated for longer, increasing from an average of nine years for those affiliated between 1985 and 1989, to twelve years for those affiliated between 1995 and 1999.

The average age of the population of authors of written material has risen over the last 30 years, a phenomenon common to all author categories: over half of affiliate authors were aged over 45 in 2013, whereas the 18-29 age group form just 4\% of this group. Whilst authors' income increases the longer they are members, reaching a median of 25,000 euros (before tax) after fifteen years of affiliation, we see that authors affiliated since the 2000 s have falling revenue prospects. The younger generations of authors show lower income levels to those of their older counterparts at the same point in their career, as compared over equal affiliation periods. Consequently, young authors are increasingly unable to make a living purely from their creative careers and are thus relying on other sources of income. These results indicate the need for an increased focus on young creatives to ensure the ongoing renewal of this profession in an unfavourable economic climate.

Directeur de la publication : Loup Wolff, chef du Département des études, de la prospection et des statistiques Responsable de la publication : Edwige Millery

\section{Retrouvez l'ensemble des publications du DEPS : http://www.culturecommunication.gouv.fr/Etudes-et-statistiques http://www.cairn.info/editeur.php?iD_EDITEUR=DEPS http://www.books.openedition.org/deps}

Le DEPS n'assurant pas de diffusion physique de ses collections de synthèse, nous vous proposons de vous informer régulièrement des parutions par message électronique.

Pour ce faire, merci de bien vouloir nous communiquer votre courriel à l'adresse contact.deps@culture.gouv.fr 
